\title{
A Universal Strategy for Organic Fluid Phosphorescence Materials
}

Siyu Sun, Jie Wang, Liangwei Ma, Xiang Ma* and He Tian

Key Laboratory for Advanced Materials and Feringa Nobel Prize Scientist Joint Research Center,

Frontiers Science Center for Materiobiology and Dynamic Chemistry, School of Chemistry and Molecular Engineering, East China University of Science and Technology, Shanghai 200237, China.

E-mail: maxiang@ecust.edu.cn

It has become an accepted approach to construct room-temperature phosphorescence (RTP) materials by suppressing the non-radiative decay process. However, there is limited success in developing fluid phosphorescence materials due to the ultrafast non-radiation relaxation of vibration and collision of molecules in fluid matrixes. In this study, a universal strategy was proposed for pure organic phosphorescent fluid materials that are able to generate effective phosphorescent emissions at both room temperature $\left(\Phi_{\mathrm{RTP}, 293 \mathrm{~K}} \sim 30 \%\right)$ and even higher temperature $\left(\Phi_{\mathrm{RTP}, 358 \mathrm{~K}} \sim \mathbf{4 . 5 3 \%}\right)$. Based on these findings, a qualitative analytical method was developed for leak detection and a quantitative analytical technique was further validated to help visually identify the heat distribution of irregular surfaces. This advancement greatly empowers the current organic phosphorescent system offering an alternative approach to determine moisture and heat from non-invasive photoluminescence emission colors. 
So far, metal-free room-temperature phosphorescence (RTP) materials ${ }^{1-14}$ has received a lot of attention, as the characteristic of large Stokes shift and long lifetime of RTP materials makes them promising in biomedical materials and photoelectric materials. The solid-state is considered the mainstream phase state of metal-free RTP materials due to the low non-radiative transition constant of organic phosphors in rigid micro-environments, while in the fluid state phase, the non-radiative transition can effectively quench RTP emissions in the fluid environment in vivo for the uncontrollable intermolecular collision and intramolecular vibration process in flowable matrixes. Thus, a general strategy based on the hydrogen-bonding interactions to construct flowable RTP materials may significantly improve the progress of RTP materials. The hydrogen-bonding interactions are considered as integral to realize a rigid micro-environment for RTP emissions. The strong hydrogen-bonding interactions are considered a key breakthrough in effectively restraining the ultrafast non-radiative decay of RTP in flowable matrixes. In this study, a universal strategy focusing on deep-eutectic-solvent-based approaches ${ }^{15,16}$ was proposed to construct flowable pure organic RTP materials. The preparation materials include commercially available organic compounds and metal-free RTP phosphor. The internal multi-hydrogen bonding can provide a rigid micro-environment to reduce non-radiative decay ${ }^{16}$. This strategy is proved very effective because fluid materials achieved a high RTP quantum yield $\left(\Phi_{\mathrm{P}} \sim 30 \%\right)$ and decent phosphorescence emissions $\left(\Phi_{\mathrm{P}} \sim 4.53 \%\right)$ even at $85{ }^{\circ} \mathrm{C}$. These properties of these fluid materials show enormous potentials in biomedical materials because of the existence of widespread soft environments full of hydrogen bonds in vivo. Additionally, a unique coorelation between temperature and photoluminescence color from $20^{\circ} \mathrm{C}$ to $85^{\circ} \mathrm{C}$ can be realized, which shows extensive application prospect in the field of visualized temperature distribution detection regardless of the morphology 
of testing objects.

Two kinds of metal-free flowable materials with moderate RTP emissions were constructed by using two organic acids, $\beta$-CD and three organic dyes. One kind of materials (including RMM, BMM, and GMM) was constructed by $\beta-\mathrm{CD}$ and malic acid (MM) in the molar ratio of 1:15 with three doping dyes (Figure 1a), 7-chlorothiochroman-4-one ${ }^{17}$, 1-bromonaphthalene ${ }^{18}$ and $\mathrm{N}$-propyl4-bromo-1,8-naphthalimide ${ }^{18}$ respectively, covering the entire visible light region $(380 \sim 780 \mathrm{~nm})^{19}$. The other kind of materials (including RCM, BCM, and $\mathbf{G C M}$ ) was constructed by $\beta$-CD and citric acid $(\mathbf{C M})$ in the same molar ratio with the same three doping dyes, respectively. The doping concentration of dyes was $0.1 \mathrm{~mol} \%$. The flowable materials thus constructed were transparent liquids (Figure 1b left), with the photographs of their RTP emissions shown in Figure $1 \mathrm{~b}$ right. Furthermore, a two-phosphor flowable material with white light emission (Commission Internationale de L'éclairage (CIE) coordinates $(0.33,0.31))$ shows the properties of homogeneous mixing RTP emissions (Figure 1e, Inset) in a certain molar ratio (1:3) through mixing 7chlorothiochroman-4-one and $N$-propyl-4-bromo-1,8-naphthalimide together in MM matrix, confirming the simplicity to obtain multi-color emission system by using these flowable materials. 

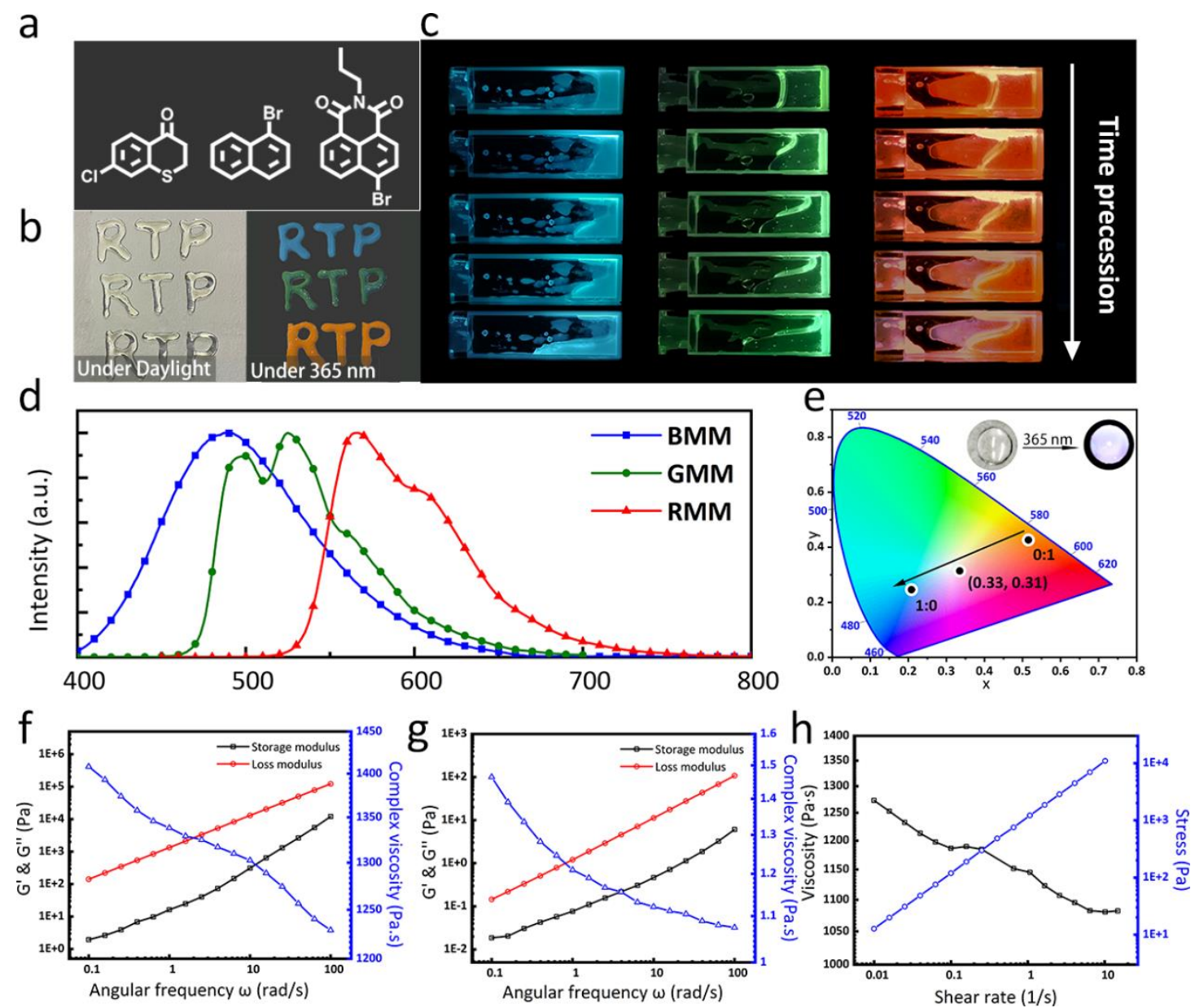

Figure 1. (a) Structure of phosphors to construct flowable materials, (b) photographs of BMM $\left(\lambda_{\mathrm{ex}}\right.$ $=365 \mathrm{~nm}), \mathbf{G M M}\left(\lambda_{\mathrm{ex}}=254 \mathrm{~nm}\right), \mathbf{R M M}\left(\lambda_{\mathrm{ex}}=365 \mathrm{~nm}\right)$ in daylight (left) and under the excitation of a portable UV lamp (right), (c) photographs for malic-acid based RTP flowable materials, (d) the delayed spectra of BMM (sky-blue line, $\lambda_{\mathrm{ex}}=360 \mathrm{~nm}$ ), GMM (green line, $\lambda_{\mathrm{ex}}=250 \mathrm{~nm}$ ), and RMM (red line, $\lambda_{\mathrm{ex}}=360 \mathrm{~nm}$ ) at $293 \mathrm{~K}$, (e) CIE 1931 chromaticity diagram of two-phosphor flowable RTP material $(0 / 1,1 / 3,1 / 0, \mathrm{~mol} / \mathrm{mol}, \quad 7$-chlorothiochroman-4-one/ $N$-propyl-4-bromo-1,8naphthalimide in MM matrix) at $293 \mathrm{~K}\left(\lambda_{\mathrm{ex}}=360 \mathrm{~nm}\right)$, Storage modulus $\left(\mathrm{G}^{\prime}\right)$ and loss modulus $\left(\mathrm{G}^{\prime \prime}\right)$ values of RMM on frequency sweep (left axis) and composite viscosity ( $\eta$ ) value of RMM on frequency sweep (right axis) at $293 \mathrm{~K}$ (f), at $393 \mathrm{~K}$ (g). (h) the viscosity-shear rate curve (black, left axis) and the stress-shear rate curve (blue, right axis) of RMM materials at $293 \mathrm{~K}$.

The malic-acid-based fluid materials were first analyzed. A rotational rheometer verified the flowable properties of malic-acid-based RTP flowable materials at different temperatures. All flowable materials were viscoelastic fluid at room temperature $\left(25^{\circ} \mathrm{C}\right.$, Figure $2 \mathrm{f}$, Figure S21-26) and with storage modulus $\left(\mathbf{G}^{\prime}\right)$ and loss modulus $\left(\mathbf{G}^{\prime \prime}\right)$ greater than zero at a high temperature $\left(100^{\circ} \mathrm{C}\right.$, 
Figure S21-26). The flowability of these materials at high temperatures became significantly stronger than at low temperatures. Equation (S1) and Equation (S2) are definition equations of $\mathbf{G}^{\prime}$ and $\mathbf{G}^{\prime \prime}$, in which $\boldsymbol{\omega}$ is the angular frequency, $\boldsymbol{t}$ is the time, and $\boldsymbol{G}(\boldsymbol{t})$ is the relaxation modulus (the function of time variation). Equation (S3) describes the relaxation time $\boldsymbol{\tau}$ of the viscoelastic flowable, which was used to characterize the dominant nature between viscosity and elasticity properties in a viscoelastic fluid. Figure S21-26 shows that the malic-acid-based flowable RTP materials were high viscosity flowable at room temperature. Compared with $\boldsymbol{\tau}$ in different flowable materials, RMM shows theoretically the highest $\boldsymbol{\tau}$ value $\left(\sim 10^{3}\right)$ with the best flowable performance. This was consistent with the experimental results. RMM showed/presented linear relationships between viscosity and angular frequency $\boldsymbol{\omega}$, differing from other nonlinear viscoelastic flowable RTP materials. The linear viscoelastic property of RMM is liable to modeling and analyzing large-scale applications.

Table 1 displays detailed photophysical data of flowable materials ( $0.1 \mathrm{~mol} \%)$ at $293 \mathrm{~K}$. Figure S1-S3 shows the delayed spectra and the prompted spectra of malic-acid-based flowable RTP materials at $293 \mathrm{~K}$. All these materials generated strong delayed emissions and relatively weak prompted emissions. The overlap of absorption spectra and excitation spectra proves that the delayed and the prompted emission share the same Franck-Condon excited state (Figure S1-S3, Figure S10-12). All delayed emissions observed from these materials were considered as RTP emissions, consistent with the reported RTP emission of these phosphors in solid rigid matrixes ${ }^{17,18}$. Exhibiting the most efficient RTP emissions $\left(\Phi_{\mathrm{RTP}, 293 \mathrm{~K}} \sim 30 \%\right)$ located at $500 \mathrm{~nm}$, the prompted emission of GMM could not be observed in both steady-state photoluminescence spectra and fluorescence spectra. RMM, instead, exhibited weak fluorescence emission and strong RTP 
emission $\left(\Phi_{\text {RTP, } 293 \mathrm{~K}} \sim 17 \%\right)$, and the color between fluorescence emission $(\sim 400 \mathrm{~nm})$ and RTP emission ( $\sim 550 \mathrm{~nm})$ was close to the complementary color in the visible region. It could be observed with unaided eyes that $\mathbf{R M M}$ still had moderate phosphorescence emissions $\left(\Phi_{\mathrm{RTP}, 358 \mathrm{~K}} \sim 4.53 \%\right)$ even at $85{ }^{\circ} \mathrm{C}$, comparable with fluorescence emissions. The relative quantum yield measurement with controlled variables methods was used to evaluate the RTP emission efficiency of the RMM at different temperatures. The temperature-dependent, steady-state photoluminescence spectra (Figure 2a) provided the evidence for calculating the relative quantum yield of the RMM, and the substantially unchanged absorption spectra (Figure S13) of the RMM before and after heating ensured the reliability of the calculated quantum yield. Figure $2 b$ shows the temperature-dependent RTP relative quantum yield variation of RMM in ambiance, and the phosphorescence relative quantum yield of RMM in $358 \mathrm{~K}$ was up to $4.53 \%$. Rarely would organic room-temperature phosphorescence materials have effective phosphorescent emissions at higher temperatures.

Table 1. Detailed photophysical properties of flowable RTP materials ( $0.1 \mathrm{~mol} \%$ phosphors) at 293 K. F: fluorescence; P: phosphorescence; RTP: room-temperature phosphorescence; PL: photoluminescence.

\begin{tabular}{ccccccc}
\hline Compound & $\boldsymbol{\lambda}_{\text {ex }}[\mathbf{n m}]$ & $\boldsymbol{\lambda}_{\text {em }}(\mathbf{F})[\mathbf{n m}]$ & $\boldsymbol{\lambda}_{\text {em }}(\mathbf{P})[\mathbf{n m}]$ & $\boldsymbol{\tau}_{\mathbf{P}}[\mathbf{m s}]$ & $\boldsymbol{\Phi}_{\mathbf{R T P}}[\boldsymbol{\%}]$ & $\boldsymbol{\Phi}_{\mathbf{P L}}[\boldsymbol{\%}]$ \\
\hline $\mathbf{B M M}$ & 360 & 445 & 490 & 3.983 & $-\mathrm{b}$ & 5.9 \\
$\mathbf{B C M}$ & 360 & 453 & 496 & 8.821 & - & 12.4 \\
$\mathbf{G M M}$ & 300 & $\mathrm{ND}^{\mathrm{a}}$ & 500,523 & 7.13 & 30.7 & 30.9 \\
$\mathbf{G C M}$ & 300 & $\mathrm{ND}$ & 500,523 & 5.68 & 20 & 20.3 \\
$\mathbf{R M M}$ & 340 & 402 & 565,608 & 4.945 & 17 & 26.8 \\
$\mathbf{R C M}$ & 340 & 401 & 567,610 & 6.023 & 16.2 & 25.6 \\
\hline
\end{tabular}

${ }^{\mathrm{a}} \mathrm{ND}$ : Not detected; ${ }^{\mathrm{b}}$ - The quantum yields of BMM and BCM were not provided in the table, because the fluorescence and phosphorescent emission peak splitting was not obvious, making it difficult to obtain reliable quantum yield data effectively.

Due to this fluid phosphorescence properties, the proposed fluid RTP materials may be 
extensively applied. With good/strong viscosity, fluid RTP materials can be effectively adhered on irregular surfaces of materials rich in hydrogen bonds, regardless of the surface morphology of the material. Meanwhile, for hydrogen-bonding-based RTP materials, fluid RTP materials have been experimentally viable to understand that extra water molecules into the hydrogen-bonding-based RTP materials can break the hydrogen-bonding-based rigid micro-environment to quench RTP emissions with basically unchanged fluorescence left ${ }^{17}$, and such properties can also be found on these flowable materials. Due to the high contrast of the fluorescence and phosphorescence of the RMM, its water-responsive properties were used to detect the qualitative impermeability of watercontaining systems with unaided eyes (Figure S29). RMM was applied to effectively seal the mouth of a rough ground bottle with a small amount of water. After 24 hours, the quenched red phosphorescence emission at its ground mouth could be observed, proving the exchange of water vapor between the bottle and the atmosphere. This property can be used to visually prevent and detect the exchange of water vapor between industrially closed systems and the environment.

Furthermore, the temperature-responsive PL emission was observed with unaided eyes. Figure 2a shows the temperature-dependent steady-state PL spectra of RMM $\left(\lambda_{\mathrm{ex}}=280 \mathrm{~nm}\right.$ to unabridged display of the PL spectra of RMM) from $293 \mathrm{~K}$ to $353 \mathrm{~K}$, where the RTP emission was dominant at 293 K. Figure 2c reveals the CIE 1931 chromaticity diagram of RMM at different temperatures, whose data were correlated to the PL spectra in Figure 2a. It can be found that the color variations of the RMM at different temperatures present an experiential and functional relationship. It is not rigorous, however, for researchers to use color to measure the temperature of environments based on the cone-cell-dependence characteristics of color (like the brightness in RGB color model et al.). Therefore, the correlated color temperature can be chosen because the CIE coordination of RMM 
at different temperatures are located on the Planck curve or the white light region with differing color temperatures, and the correlated color temperature (CCT) index Mired value can be used to measure the relationship between the environmental temperature and emission properties. For the interlinkage between CIE 1931 data and CCT value, Equation (S4) and Equation (S5) were experiential equations to calculate the experimental CCT value of PL emissions at differing temperatures ${ }^{20}$.

Polynomial fitting was used to fit the functional relationship of experimental Mired value (Mired $=\frac{10^{6}}{\mathrm{CCT}}$ ) and environmental temperature, in which Equation (S6) served as the empirically fitted equation (Figure S28). Table S9 illustrates the experimental temperature, the fitted calculated temperature and their errors $(<5 \%)$. It was assumed that the experimental Mired value for RMM can be used to estimate the current environmental temperature in a steady state. Figure $2 \mathrm{e}$ shows the photographs of color change with temperature. Accordingly, RMM showed great application potentials in real-time temperature response probes due to the high sensitivity of their RTP intensity and flowable ability in overlooking the surface of topography objects. Also, RMM was applied on the surface of a ceramic plate on a digital heating magnetic stirrer to test its sensitivity to the change in temperature through visual qualitative observation (Figure S29). The unevenly heated condition of the ceramic plate in real-time and the ensuing gradual and evenly heated process along with the time (Figure S29) can be observed with unaided eyes. As a highly temperature-sensitive probe based on RTP, RMM can be effectively attached to any surface of solid materials to detect the change in local temperature due to its flowable properties and extensive adhesion to solid materials. Based on this finding, a method based on RMM fluid materials was proposed to link temperature and color temperature so as to achieve efficient quantitative detection of temperature distribution. A camera 
can be used to photograph the color and color temperature change of the fluid surface, and specific temperature distributions of the object surface and the time-resolved temperature change can further be quantified by analyzing the color and color temperature change combined in the program, making a quantitative study of temperature distributions of the object available (refer to the description of this quantitative analysis in Supporting Information). This system is expected to be applied in fields like qualitative temperature distribution analysis and hydromechanics analysis.
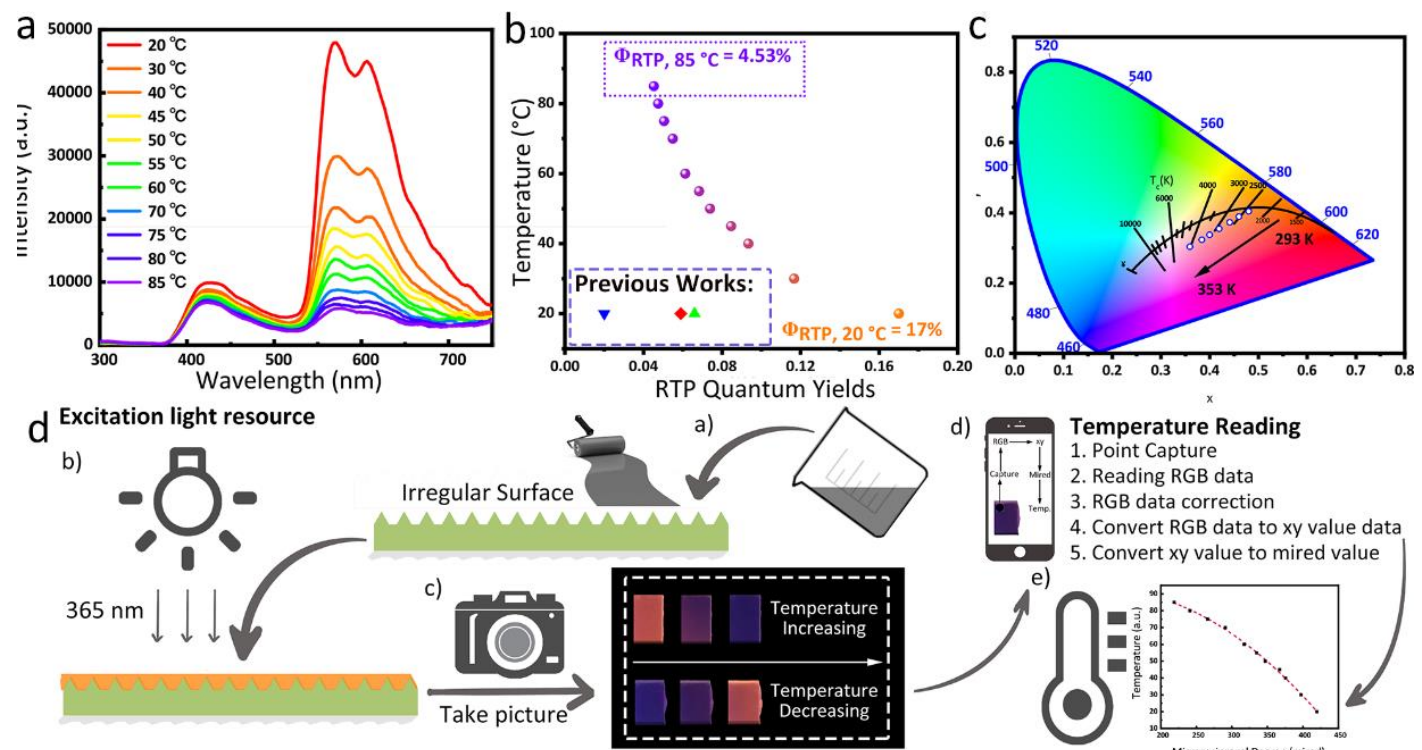

\section{Irregular Surface}

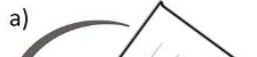

e
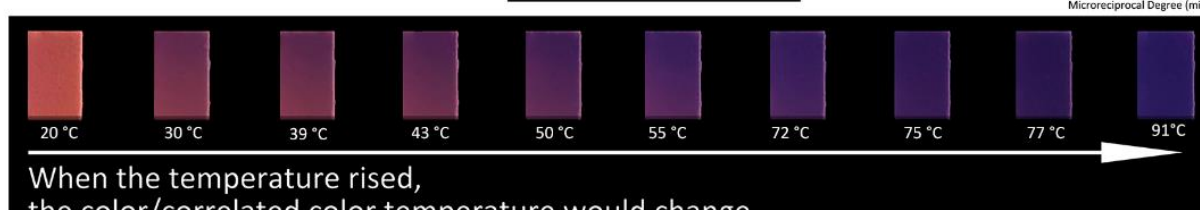

the color/correlated color temperature would change.

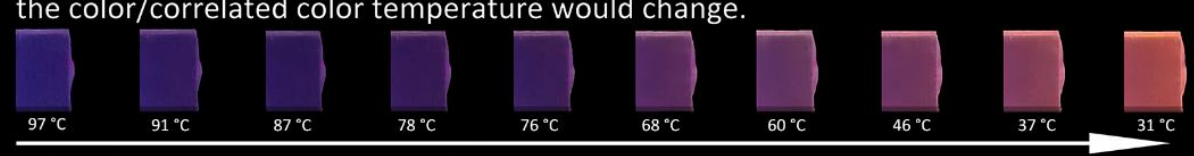

When the temperature returned to room temperature,

the color/correlated color temperature returns to original state

Figure 2 (a) Steady-state photoluminescence spectra of RMM at different temperatures (20 minutes per gradient for stabilization of temperature ); (b) The relative RTP quantum yield curve of RMM with an increase in temperature (purple and orange spheres); blue triangle, red square and green triangle refer to the RTP data from published results of NBI derivatives in different matrixes 19,21,22;

(c) RMM color coordinate chart of CIE under differing temperature conditions (insert scale: color temperature scale, temperature gradient: $10 \mathrm{~K}$ ); (d) Schematic illustration of temperature quantitative detection process by RMM; (e) Photograph of RMM coated plate heated from $20^{\circ} \mathrm{C}$ to $100^{\circ} \mathrm{C}$ on a heating plate. 
In this study, a reliable and convenient large-scale preparation strategy was proposed to construct metal-free fluid RTP materials with high-temperature tolerance, which was also proved general and original in preparing flowable metal-free RTP materials with moderate RTP emissions ( $\sim 30 \%)$. The phosphorescence of the RMM demonstrates strong temperature tolerance even at a high temperature of $85{ }^{\circ} \mathrm{C}\left(\Phi_{\mathrm{RTP}}, 358 \mathrm{~K} \sim 4.53 \%\right)$. Based on these properties, a visualized wetness/temperature-responsive system was designed, in which the wetness-responsive properties were used to detect impermeability of special systems, and the temperature-responsive properties were applied toalalyze qualitatively the temperature distribution on the surface of an object. These flowable phosphorescent materials with strong hydrogen bonds present extensive potentials for large-scale preparation and applications, which also show great potentials in biomedical materials due to widespread fluid environments with hydrogen bonds in vivo. Last but not least, this approach may further extend the application of metal-free RTP materials at higher temperatures.

\section{Acknowledgements}

We acknowledge the financial support from NSFC (21788102, 22020102006 and 21871083), project support by the Shanghai Municipal Science and Technology Major Project (Grant No.2018SHZDZX03), Program of Shanghai Academic/Technology Research Leader (20XD1421300), 'Shu Guang' project supported by Shanghai Municipal Education Commission and Shanghai Education Development Foundation (19SG26), and the Innovation Program of Shanghai Municipal Education Commission (2017-01-07-00-02-E00010). The authors thank Dr. B. Ding and Mr. Z. Huang in our lab for helpful discussion, Mr. Y. Lu in Shanghai University for helpful discussion on rheology, and Dr. X. Rao from ECUST for helpful manuscript polishment. 


\section{Author contributions}

S. S. and X. M. proposed the project and designed the experiments. S. S. carried out all the preparation of the materials, photophysical experiments. S. S., X. M. and H. T. co-wrote the manuscript. J. W. and L. M. provided some insightful suggestions on the manuscript. X. M. and H. T. supervised the project. All authors discussed the results and participated in analyzing the experimental results.

\section{Competing interests}

X. M., S. S. and H. T. are inventors on a provisional patent application related to this work that has been filed by the East China University of Science and Technology (Application no. 202110387050.X, date: 12 Apr 2021). The authors declare no other competing interests.

\section{Reference:}

1 Ma, X., Wang, J. \& Tian, H. Assembling-Induced Emission: An Efficient Approach for Amorphous Metal-Free Organic Emitting Materials with Room Temperature Phosphorescence. Acc. Chem. Res. 52, 738-748, (2019).

2 Zhang, T. et al. Molecular Engineering for Metal-Free Amorphous Materials with RoomTemperature Phosphorescence. Angew. Chem. Int. Ed. 59, 11206-11216, (2020).

3 Wang, X. et al. Organic phosphors with bright triplet excitons for efficient X-ray-excited luminescence. Nature Photon. 187-192, (2021).

4 Gong, Y. et al. Achieving Persistent Room Temperature Phosphorescence and Remarkable Mechanochromism from Pure Organic Luminogens. Adv. Mater. 27, 6195-6201, (2015).

5 Wang, X. F. et al. Pure Organic Room Temperature Phosphorescence from Unique MicelleAssisted Assembly of Nanocrystals in Water. Adv. Funct. Mater. 30, 1907282, (2020).

6 Zhou, B. \& Yan, D. Hydrogen-Bonded Two-Component Ionic Crystals Showing Enhanced Long-Lived Room-Temperature Phosphorescence via TADF-Assisted Forster Resonance Energy Transfer. Adv. Funct. Mater. 29, 1807599, (2019).

7 Zhao, W. J., He, Z. K. \& Tang, B. Z. Room-temperature phosphorescence from organic aggregates. Nature Rev. Mater. 869-885, (2020).

8 Wang, Y. et al. Persistent organic room temperature phosphorescence: what is the role of molecular dimers? Chem. Sci. 11, 833-838, (2020).

9 Kamatsuki, T., Bhattacharjee, I. \& Hirata, S. The Substituent-Induced Symmetry-Forbidden 
Electronic Transition Allows Significant Optical Limiting under Weak Sky-Blue Irradiance. $J$. Phys. Chem. Lett. 11, 8675-8681, (2020).

$10 \mathrm{Gu}$, L. et al. Colour-tunable ultra-long organic phosphorescence of a single-component molecular crystal. Nature Photon. 13, 406-411, (2019).

11 Chen, C. J. et al. Carbazole isomers induce ultralong organic phosphorescence. Nature Mater. 175-180, (2020).

12 Bhattacharjee, I. \& Hirata, S. Highly Efficient Persistent Room-Temperature Phosphorescence from Heavy Atom-Free Molecules Triggered by Hidden Long Phosphorescent Antenna. Adv. Mater. 32, e2001348, (2020).

Yu, Y. et al. Room-Temperature-Phosphorescence-Based Dissolved Oxygen Detection by CoreShell Polymer Nanoparticles Containing Metal-Free Organic Phosphors. Angew. Chem. Int. Ed. 56, 16207-16211, (2017).

He, Z. et al. Achieving Persistent, Efficient, and Robust Room-Temperature Phosphorescence from Pure Organics for Versatile Applications. Adv. Mater. 31, e1807222, (2019).

Dong, S. et al. Structural water as an essential comonomer in supramolecular polymerization. Sci. Adv. 3, eaao0900, (2017).

Wu, S., Cai, C., Li, F., Tan, Z. \& Dong, S. Deep Eutectic Supramolecular Polymers: Bulk Supramolecular Materials. Angew. Chem. Int. Ed. 59, 11871-11875, (2020).

17 Ma, L., Sun, S., Ding, B., Ma, X. \& Tian, H. Highly Efficient Room-Temperature Phosphorescence Based on Single-Benzene Structure Molecules and Photoactivated Luminescence with Afterglow. Adv. Funct. Mater. 2010659, (2021).

Li, D. et al. Amorphous Metal-Free Room-Temperature Phosphorescent Small Molecules with Multicolor Photoluminescence via a Host-Guest and Dual-Emission Strategy. J. Am. Chem. Soc. 140, 1916-1923, (2018).

Starr, C., Evers, C. A. \& Starr, L. Biology : concepts and applications. 8th edn, (Brooks/Cole Thomson, 2011).

20 McCamy, C. S. Correlated color temperature as an explicit function of chromaticity coordinates. Color Res. Appl. 17, 142-144, (1992).

21 Goudappagouda et al. Paintable Room-Temperature Phosphorescent Liquid Formulations of Alkylated Bromonaphthalimide. Angew. Chem. Int. Ed. 58, 2284-2288, (2019). Yao, X. et al. Room-Temperature Phosphorescence Enabled through Nacre-Mimetic Nanocomposite Design. Adv. Mater. 33, 2005973, (2021). 